\title{
RELACIÓN ENTRE ACTIVIDAD FÍSICA Y OBESIDAD EN ESCOLARES DE PRIMARIA DEL NORTE DE MÉXICO
}

\section{RELATIONSHIP BETWEEN PHYSICAL ACTIVITY AND OBESITY IN ELEMENTARY SCHOOL CHILDREN IN MEXICO NORTHERN}

Recibido el 10 de noviembre de 2020 / Aceptado el 10 de enero de 2021 / DOI: 10.24310/riccafd.2021.v10i1.10650 Correspondencia: Salvador Jesús López Alonzo. Facultad de Ciencias de la Cultura Física, Universidad Autónoma de Chihuahua, México. Periférico de la Juventud y Circuito Universitario, S/N. Col. Paseos de Chihuahua, C.P. 31125. Teléfono: 0526141589900 E-mail: salopez@uach.mx

López-Alonzo, S.J. ${ }^{1 A-F} ;$ Gastelúm Cuadras, G. ${ }^{2 A-D}$; Islas Guerra, S.A. ${ }^{3 B C}$; Chávez Erives I.A. ${ }^{4 B C}$ y Orona Escapite A. ${ }^{5 B C}$

${ }^{1}$ Profesor Investigador (Doctor en Educación) de tiempo completo, Universidad Autónoma de Chihuahua, Facultad de Ciencias de la Cultura Física, Chihuahua, México, salopez@uach.mx

${ }^{2}$ Profesor Investigador (Doctor en Actividad Física y Salud) de tiempo completo, Universidad Autónoma de Chihuahua, Facultad de Ciencias de la Cultura Física, Chihuahua, México, gastelum@uach.mx

${ }^{3}$ Docente hora clase (Doctor en Ciencias de la Actividad física y Deporte), Universidad Autónoma de Chihuahua, Facultad de Ciencias de la Cultura Física, Chihuahua, México, saislas@uach.mx

${ }^{4}$ Docente hora clase (Doctor en Ciencias de la Actividad física y Deporte), Universidad Autónoma de Chihuahua, Facultad de Ciencias de la Cultura Física, Chihuahua, México, achavez@uach.mx

${ }^{5}$ Docente de medio tempo (Maestra en Atención a Poblaciones Especiales), Universidad Autónoma de Chihuahua, Facultad de Ciencias de la Cultura Física, Chihuahua, México, aorona@uach.mx

\section{Responsabilidades}

${ }^{A}$ Diseño de la investigación. ${ }^{B}$ Recolector de datos. ${ }^{C}$ Redactor del trabajo. ${ }^{D}$ Tratamiento estadístico. EApoyo económico. Fldea original y coordinador de toda la investigación

\section{RESUMEN}

El objetivo del presente estudio fue analizar la relación entre los niveles de actividad física y obesidad en escolares de primaria pertenecientes a una escuela pública estatal de la ciudad de Chihuahua, México. Se realizó un estudio descriptivo, correlacional y transversal, se seleccionó una muestra por conveniencia, 326 escolares de 9 años (178 niñas y 148 niños). Los niveles de Actividad Física (AF) fueron estimados mediante una entrevista, se utilizó el "The Godin-Shephard Leisure-Time Physical Activity Questionnaire" (GSLTPAQ) y para identificar la obesidad se utilizó el Índice de Masa Corporal (IMC) y la Circunferencia de Cintura (CC). El análisis estadístico nos mostró correlaciones bilaterales de Pearson negativas con significancia entre la AF vigorosa y el IMC $(r=-.139, p \leq 0.050)$, así como entre la CC $(r=-.150$, 
$p \leq 0.010$ ). Además los escolares con obesidad mostraron que realizan significativamente menos AF vigorosa por semana, al compararlos con los que presentan sobrepeso $(p \leq 0.050)$, y los que presentan normopeso $(p \leq 0.010)$. Los resultados indican que escolares con mayor IMC y CC, así como los que presentan obesidad realizan menos AF vigorosa por semana de forma significativa. Por lo que es necesario que se promuevan actividades físicas vigorosas dentro y fuera de la escuela en este tipo de poblaciones, con el fin de prevenir la obesidad.

\section{PALABRAS CLAVE}

actividad física, escolares, obesidad.

\section{ABSTRACT}

The objective of this study was to analyze the relationship between levels of physical activity and obesity in elementary school students belonging to a state public school in the city of Chihuahua, Mexico. A descriptive, correlational and cross-sectional study was carried out, a sample was selected for convenience, 326 schoolchildren of 9 years of age (178 girls and 148 boys). The levels of Physical Activity (PA) were estimated through an interview, using the "The Godin-Shephard LeisureTime Physical Activity Questionnaire" (GSLTPAQ) and to identify obesity the Body Mass Index (BMI) and the Waist Circumference (WC). The statistical analysis showed us negative bilateral Pearson correlations with significance between vigorous PA and BMI $(r=-.139, p \leq 0.050)$, as well as between WC $(r=-.150, p \leq 0.010)$. In addition, schoolchildren with obesity showed that they perform significantly less vigorous PA per week, when compared to those who are overweight $(p \leq 0.050)$, and those with normal weight $(p \leq 0.010)$. The results indicate that schoolchildren with higher BMI and WC, thus those with obesity significantly perform less vigorous PA per week. Consequently, it is necessary to promote vigorous physical activities inside and outside of school in this type of population, in order to prevent obesity.

\section{KEY WORDS}

Physical activity, schoolchildren, obesity.

\section{INTRODUCCIÓN}

La AF es considerada como cualquier movimiento musculo esquelético voluntario, que ocasiona un gasto calórico por encima de lo basal ${ }^{1}$. 
La inactividad física es una de las problemáticas a nivel mundial que impacta en la salud de la población, esta se vincula a problemas de sobrepeso, obesidad, así como a enfermedades no transmisibles ${ }^{1,2}$. Se estima que, el $81 \%$ de la población de adolescentes en edad escolar en el mundo no realizan suficiente $A^{1,2}$. El problema de la inactividad física es multifactorial; los avances tecnológicos, la urbanización, la falta de acceso a centro deportivos, parques, así como la inseguridad social, contaminación entre otros factores, ha contribuido a la disminución de los niveles de AF de la población en general ${ }^{1-4}$.

Según las recomendaciones mundiales, de AF para la salud, los niños, niñas y adolescentes deben realizar al menos 60 minutos diarios de AF de intensidad moderada a vigorosa. Además, en estos debe incluir actividades en donde se fortalezca el sistema musculo esquelético y mejoren flexibilidad ${ }^{1,3-5}$.

Por otra parte, las políticas de salud pública indican que la promoción de la AF en escolares debe ser clave para currículos educativos, desde la Educación Física como base, pero, en colaboración con los diferentes sectores públicos y privados de la sociedad ${ }^{4-6}$.

Existen estudios epidemiológicos en México que estiman que cerca del $80 \%$ de los niños entre 10 y 14 años de edad son considerados inactivos físicamente ${ }^{6,7}$. Además, el $32 \%$ de los escolares entre 5 y 11 años de edad presentan sobrepeso y obesidad infantil, lo que es considerado como una problemática de salud pública, por lo que se podría vincular a la inactividad física $^{6-8}$. En un estudio realizado por Hall-López et al. ${ }^{9}$, en México con escolares durante las clases de EF, mostro que la AF durante las sesiones de esta asignatura es de baja intensidad, además, con dos sesiones por semana el estímulo de la AF es muy pobre y no alcanza para cumplir con las recomendaciones que establece la OMS en materia de AF para la salud.

Identificar los niveles de actividad que practican los niños y adolescentes, es de relevancia para la salud en los escolares, por lo que pueden ser de suma importancia para poder establecer medidas pertinentes, en el diseño de programas integrales con un enfoque holístico, para promover e implementar estilos de vida activos y saludables ${ }^{10-12}$. El cuestionario GSLTPAQ, ha sido uno de los más utilizados en diversos estudios, pues resulta un instrumento relativamente fácil de utilizar, este cuestionario toma en cuenta los niveles de AF ligera, moderada y vigorosa que realizan las personas, además ha sido validado y utilizado en diferentes poblaciones ${ }^{12-14}$.

El propósito principal del presente estudio fue analizar la relación entre la AF y la obesidad en escolares de primaria, se utilizó el GSLTPAQ para identificar los niveles de AF, por otra parte, se utilizó como indicadores de obesidad el IMC y la Circunferencia de Cintura (CC) 


\section{MATERIAL Y MÉTODOS}

La presente investigación fue un diseño metodológico de tipo transversal, descriptivo y correlacional, se seleccionó por conveniencia, una escuela de nivel primaria, del subsistema estatal de educación pública en la ciudad de Chihuahua, México. Se analizaron 326 escolares (178 niñas y 148 niños) de edades entre 6 y 12 años, quienes participaron voluntariamente, con autorización de los padres o tutores, a través de firma de una carta de consentimiento informado, previamente se solicitó permiso a las autoridades correspondientes y se informó del proyecto.

Para estimar los niveles de AF se usó el cuestionario GSLTPAQ, con el cual se obtuvo la información necesaria, los sujetos reportaron la AF que realizan normalmente en una semana, así como el tiempo y la cantidad de veces que realiza diferentes tipos de actividades físicas en su tiempo libre. Para la aplicación de este cuestionario se requirió capacitación previa y personal calificado con conocimientos conceptuales de AF vigorosa, moderada y ligera. Fue necesario entrevistar a los escolares de forma personal, durante la clase de Educación Física, la entrevista duraba aproximadamente entre 2 y 5 minutos, se contó con tres entrevistadores para este proyecto.

Para poder interpretar claramente los diferentes niveles de AF (moderada, ligera o vigorosa) que realizan los escolares, así como el tiempo y número de veces por semana que la practican, fue necesario realizar una entrevista guiada a los escolares.

A continuación, se presenta la fórmula que utiliza el GSLTPAQ, para clasificación del "nivel de AF semanal" = $(A F$ vigorosa $\times 9)+(A F$ moderada $x 5)+($ AF ligera $x 3)$. Con resultados de 24 unidades o más, se considera una persona activa con beneficios sustanciales. De 14 a 23 unidades, se considera moderadamente activo con algunos beneficios. Si se obtiene menos de 14 unidades, se considera insuficientemente activo con muy pocos beneficios.

En el caso de la antropometría, para medir el peso corporal, los escolares se subieron a una báscula digital Tanita Fitscan BF-679F, sin calzado, con uniforme deportivo. Para medir estatura se utilizó un Estadímetro SECA 206, se colocaban a los escolares de espaldas para usar el plano de Frankfort. Para la CC se utilizó una cinta antropométrica Lufkin, se colocó a los escolares en un banco antropométrico, se midió la cintura. Específicamente se registró la medida obtenida del punto medio entre la cresta iliaca y la última costilla, esta medida, se utilizó como un indicador de obesidad central, el cual se relaciona como un factor de riesgo metabolico ${ }^{15}$. La recolección de datos se llevó a cabo durante las sesiones de Educación Física en los meses de agosto a noviembre del 2019. 
Para el análisis de los datos, se utilizó el Paquete Estadístico de Ciencias Sociales (SPSS) IBM Versión 25 para Windows. Con el cual se pudo obtener descriptivos generales y de AF de la muestra de estudio. Se realizaron medidas de frecuencia para establecer porcentajes de sobrepeso, obesidad y para la clasificación de AF. Para analizar las diferencias, se realizó un modelo lineal general de comparación múltiple con pruebas post hoc. También, para conocer la relación entre la AF y obesidad de los escolares, se realizaron correlaciones Bivariadas de Pearson entre AF vs IMC, AF vs CC.

\section{RESULTADOS}

En la tabla uno se muestran las características generales de la muestra del presente estudio (media y desviación estándar) para sexo masculino, femenino y el total, se encontraron valores promedio muy similares entre estos grupos, sin diferencias significativas.

Tabla 1. Descriptivos de las características generales de la muestra.

\begin{tabular}{lllllll}
\hline \multirow{2}{*}{ Características } & \multicolumn{2}{c}{ Niñas $(\mathrm{n}=178)$} & \multicolumn{2}{c}{ Niños $(\mathrm{n}=148)$} & \multicolumn{2}{l}{ Total $(\mathrm{n}=326)$} \\
\cline { 2 - 7 } & Media & DE & Media & DE & Media & DE \\
\hline Edad (años) & 9.1 & 2.0 & 9.0 & 2.0 & 9.1 & 2.0 \\
Estatura (cm) & 132.5 & 11.8 & 132.2 & 11.5 & 132.4 & 11.6 \\
Peso (kg) & 32.0 & 10.4 & 32.3 & 10.6 & 32.1 & 10.4 \\
Cintura (cm) & 61.3 & 9.4 & 62.6 & 10.6 & 61.9 & 10.0 \\
IMC (kg/m2) & 17.8 & 3.3 & 18.1 & 4.0 & 17.9 & 3.6 \\
\hline
\end{tabular}

DE=Desviación Estándar, IMC=Índice de Masa Corporal.

En la tabla dos se observan porcentajes de las clasificación según el IMC de ambos sexos y el total de la muestra del presente estudio. El sobrepeso y la obesidad muestran un $29.2 \%$ para la muestra total de estudio, por otra parte la obesidad es mayor en los niños con un $16.2 \%$ al comparar con las niñas que presentan un 9.6\%. La clasificación del IMC se realizó según el Food and Nutrition Technical Assistance III ${ }^{16}$.

Tabla 2. Medidas de frecuencia y porcentajes de sobrepeso y obesidad según el IMC.

\begin{tabular}{lllllll}
\hline \multirow{2}{*}{ Clasificación IMC } & \multicolumn{2}{c}{ Niñas $(\mathrm{n}=\mathbf{1 7 8})$} & \multicolumn{2}{c}{ Niños $(\mathrm{n}=148)$} & \multicolumn{2}{c}{ Total $(\mathrm{n}=\mathbf{3 2 6})$} \\
& FR & $\mathbf{\%}$ & FR & $\%$ & FR & $\%$ \\
\hline Desnutrición & 5 & 2.8 & 5 & 3.4 & 10 & 3.1 \\
Normal & 126 & 70.8 & 95 & 64.2 & 221 & 67.8 \\
\hline
\end{tabular}




\begin{tabular}{lllllll}
\hline \multirow{2}{*}{ Clasificación IMC } & \multicolumn{2}{c}{ Niñas $(\mathbf{n}=\mathbf{1 7 8})$} & \multicolumn{2}{l}{ Niños $(\mathbf{n}=\mathbf{1 4 8})$} & \multicolumn{2}{c}{ Total $(\mathbf{n = 3 2 6})$} \\
\cline { 2 - 8 } & FR & \% & FR & \% & FR & $\%$ \\
\hline Sobrepeso & 30 & 16.9 & 24 & 16.2 & 54 & 16.6 \\
Obesidad & 17 & 9.6 & 24.0 & 16.2 & 41 & 12.6 \\
IMC=Índice de Masa Corporal, FR= Frecuencias & & & & \\
\hline
\end{tabular}

En la tabla tres se pueden observar los descriptivos (media y desviación estándar) de la AF reportada en el cuestionario aplicado para sexo masculino, femenino y el total, los resultados no presentaron diferencias significativas entre niños y niñas.

Tabla 3. Descriptivos de AF reportada en el cuestionario.

\begin{tabular}{|c|c|c|c|c|c|c|}
\hline \multirow{2}{*}{ Clasificación de AF } & \multicolumn{2}{|c|}{ Niñas (n=178) } & \multicolumn{2}{|c|}{$\operatorname{Niños}(n=148)$} & \multicolumn{2}{|c|}{ Total $(n=326)$} \\
\hline & Media & DE & Media & DE & Media & DE \\
\hline AF Vigorosa (veces/semana) & 0.93 & 1.5 & 1.1 & 1.7 & 1.02 & 1.6 \\
\hline AF Moderada (veces/semana) & 1.01 & 1.7 & 0.9 & 1.6 & 0.95 & 1.6 \\
\hline AF Ligera (veces/semana) & 0.99 & 1.9 & 1.1 & 1.9 & 1.04 & 1.9 \\
\hline Total de score & 16.38 & 12.5 & 18.0 & 13.4 & 17.09 & 12.9 \\
\hline
\end{tabular}

En la figura uno se muestran las categorías en porcentajes de los niveles de AF que realizan los niños y niñas que participaron el en presente estudio, así como del total de la muestra. La AF se estimó con el GSLTPAQ.

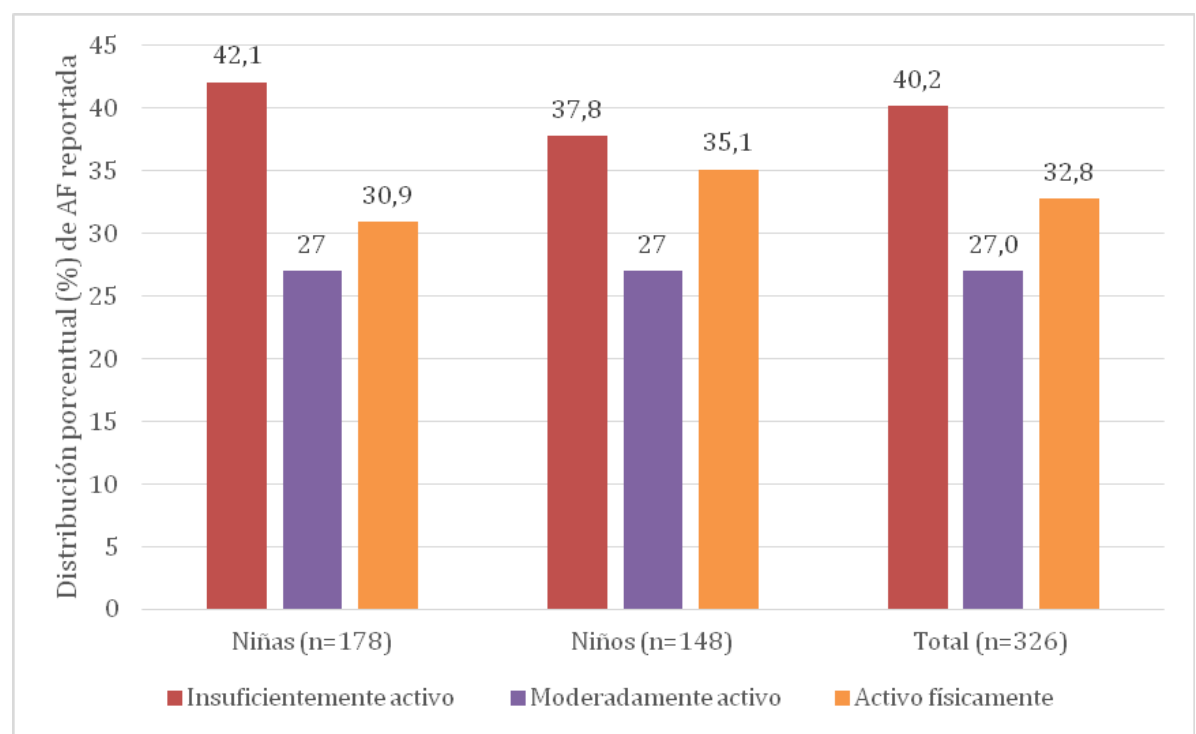

Figura 1. Distribución porcentual de AF por categorías reportada de la muestra 
En la tabla cuatro se muestra (media y desviación estándar) la AF que realizan los escolares con diferentes categorías (normo peso, sobrepeso y obesidad), así como el valor de (p). Al hacer comparaciones entre las diferentes categorías se observaron diferencias significativas en la AF vigorosa y en el puntaje total, entre los escolares con obesidad y los que no presentan.

Tabla 4. Comparaciones múltiples del estatus de peso corporal y AF con pruebas post hoc para identificar diferencias significativas.

\begin{tabular}{llllll}
\hline Clasificación AF & NP $(n=221)$ & SP $(n=54)$ & OB $(n=41)$ & NP vs OB & SP vs OB \\
Veces/semana & $\mathbf{M} \pm D E$ & $\mathbf{M} \pm D E$ & $\mathbf{M} \pm D E$ & valor $(\mathbf{p})$ & valor $(\mathbf{p})$ \\
\hline AF Vigorosa & $1.07 \pm 1.6$ & $1.02 \pm 1.7$ & $0.39 \pm 1$ & $0.010^{*}$ & $0.050^{*}$ \\
AF Moderada & $1 \pm 1.7$ & $1.2 \pm 1.8$ & $0.76 \pm 1.3$ & 0.472 & 0.150 \\
AF Ligera & $1.2 \pm 2.1$ & $0.8 \pm 1.5$ & $0.83 \pm 1.4$ & 0.271 & 0.932 \\
Total de score & $18 \pm 12.8$ & $17.8 \pm 13.5$ & $9.78 \pm 9.5$ & $0.001^{*}$ & $0.015^{*}$ \\
\hline
\end{tabular}

$\mathrm{AF}=$ Actividad Física, $\mathrm{NP}=$ Normo Peso, $\mathrm{SP}=$ Sobre Peso, $\mathrm{OB}=\mathrm{Obesidad}, \mathrm{M}=$ Media, $\mathrm{DE}=$ Desviación Estándar.

En la tabla cinco se observan las correlaciones entre los diferentes niveles de AF, la CC y el IMC de los escolares del presente estudio. Al correlacionar la AF vigorosa y el score final con los indicadores antropométricos se muestra una correlación negativa significativa.

Tabla 5. Correlación entre AF e indicadores de obesidad.

\begin{tabular}{llllll}
\hline Correlaciones & $\mathrm{IMC}\left(\mathrm{Kg} / \mathrm{m}^{2}\right)$ & AF Vigorosa & AF Moderada & AF Ligera & score final \\
\hline Cintura $(\mathrm{cm})$ & $.810^{* *}$ & $-.150^{* *}$ & 0.047 & -0.092 & $-.175^{* *}$ \\
$\mathrm{IMC}\left(\mathrm{Kg} / \mathrm{m}^{2}\right)$ & & $-.139 *$ & 0.048 & -0.054 & $-.146^{* *}$ \\
AF Vigorosa & & & $-.302^{* *}$ & $-.254^{* *}$ & $.794^{* *}$ \\
AF Moderada & & & $-.231^{* *}$ & $.199 * *$ \\
AF Ligera & & & & 0.013 \\
\hline
\end{tabular}

IMC=Índice de Masa Corporal, AF=Actividad Física, **La correlación es significativa en el nivel 0,01 (bilateral), * La correlación es significativa en el nivel 0,05 (bilateral).

En general los resultados expuestos en este estudio muestran una presencia impórtate de sobrepeso y obesidad, así como un porcentaje impórtate de escolares clasificados como insuficientemente activos. Además de encontrar correlaciones entre AF vigorosa vs IMC y AF vigorosa vs CC, se encontraron diferencias significativas entre la AF vigorosa que realizan los escolares con obesidad y los que no presentan obesidad. 


\section{DISCUSIÓN}

Los escolares del presente estudio muestran niveles de sobrepeso y obesidad de $29.2 \%$, lo cual es consistente al compararlos con el $29.5 \%$ reportado por región norte del país en la Encuesta Nacional de salud y nutrición de medio camino 7,8 . En esta misma encuesta, se observa un promedio nacional de $33.2 \%$, por lo que la prevalencia de sobrepeso y obesidad en el presente estudio es menor a la media nacional mexicana.

Por otra parte los niveles de AF no presentan diferencias al comparar niños vs niñas, por lo se puede deducir que ambos sexos realizan un número similar de veces por semana de AF. En contraste con otro estudio realizado por Alonso et al. ${ }^{17}$, en el cual si existen diferencias significativas, al ser mayor los niveles de AF que practican los niños, sin embargo, estas diferencias se obtienen al utilizar otros instrumentos, como calorimetría y otros cuestionarios, así como, una muestra de niños de seis años y españoles. Por tal motivo es posible que en el presente estudio, los datos no sean consistentes con el estudio antes mencionado.

En otro estudio publicado por García et al. ${ }^{18}$, muestra que solo un $8 \%$ de los escolares presentó un alto nivel de AF y el $28 \%$ que tenía un nivel inferior (sedentarios). En contraste con el presente estudio, en el que $40.2 \%$ se considera insuficientemente activo o sedentario y un $32.8 \%$ físicamente activo, de igual forma el cuestionario utilizado en ambos estudios es diferente y la inconsistencia puede deberse a las características de los cuestionarios.

En un estudio publicado por Ayala-Guzmán et al. ${ }^{19}$, hecho escolares mexicanos de entre 8 y 12 años de edad, se encontró que la estimación de AF ligera, moderada y vigorosa a partir del uso de acelerómetro, es muy parecida entre los escolares con peso normal, sobrepeso y obesidad. Los resultados contrastan con el presente estudio, en donde se observa que los escolares con obesidad realizan menos AF vigorosa de forma significativa. Además, se puede observar que los escolares realizan más AF ligera por semana y menos vigorosa, pero, aun sin cumplir con los mínimos recomendados ${ }^{1,9}$, por lo que los resultados de la presente investigación, son consistente con la mayoría de los estudios realizados en niños y niñas en edad escolar ${ }^{17-19}$. Es importante destacar que en la mayoría de auto reportes o cuestionarios de AF que se realizan a los escolares pueden ser poco confiables, con tendencias a sobrestimar u subestimar depende sea el caso ${ }^{17-19}$. Por tal motivo en el presente estudio se decidió hacer una entrevista guiada a los escolares por un profesional capacitada en $\mathrm{AF}$, de tal forma que se puedan ofrecer resultados con más confiabilidad.

Por otra parte las diferencias significativas entre los escolares que presentan obesidad observadas en el presente estudio, muestran 
consistencia con otro estudio publicado por Pope et al. ${ }^{20}$, donde se encontró que el porcentaje de tiempo al que le dedican a la AF los niños y niñas con peso normal es mayor de forma significativa con el que practican los niños y niñas con obesidad. Por lo que la promoción de la AF debe ser de suma importancia para los niños y niñas en edad escolar, de tal forma que impacte en la disminución de sobrepeso y obesidad.

Las correlaciones encontradas en el presente estudio muestran que existe una correlación negativa entre la AF y los indicadores de obesidad (IMC y CC), al mostrar que los niños que reportan menos AF, son los que presentan mayor obesidad. Resultados similares se encontraron en un estudio hecho por Czyż et al. ${ }^{21}$ en escolares de Polonia, en donde las correlaciones negativas entre el $\mathrm{VO}_{2}$ max e indicadores antropométricos como el porcentaje de grasa corporal $(\% \mathrm{GC})$ y el IMC reflejan que los escolares que realizan menos $\mathrm{AF}$ o que presentan menos $\mathrm{VO}_{2}$ max, son los que presentan mayor \%GC y mayor IMC.

Los resultados del presente estudio pueden tomarse como consistentes con lo publicado por Morente-Oria ${ }^{22}$, en donde concluye que es necesaria la AF vigorosa para mejores resultados en el combate de la obesidad en el ámbito escolar. Adicionalmente la presente investigación muestra evidencia en la cual, la AF vigorosa puede ser de suma importancia para prevenir la obesidad en los escolares, además se puede inferir que es necesaria la promoción de este tipo de AF dentro y fuera de la escuela, así como durante las clases de Educación Física. Sin duda, el identificar los niveles de $\mathrm{AF}$ son de gran importancia para poder planear estrategias pertinentes en los escolares y promover estilos de vida activos y saludables, sin embargo, es necesario identificar los instrumentas y los procedimientos más convenientes para el contexto y población.

\section{REFERENCIAS BIBLIOGRÁFICAS}

1. Organización Mundial de la Salud (OMS). Plan de acción mundial sobre actividad física 2018-2030: personas más activas para un mundo más sano. (2019), Disponible en: https://apps.who.int/iris/handle/10665/327897

2. OMS, Diez datos sobre la actividad física. 2017; Disponible en: https://www. who.int/features/factfiles/physical activity/es/

3. López-Alonzo SJ, Rivera-Sosa JM, Hernández-Gutiérrez PZ, et al. Relación entre fuerza muscular y estado de nutrición en escolares mexicanos. Rev Mex Pediatr. 2019; 86 (5):185-189. doi:10.35366/SP195D

4. Department of Health U, Services H, for Disease Control C, National Center for Chronic Disease Prevention and Health Promotion. Increasing physical education and physical activity: A frameworks for school. 2017, Disponible en: https:// www.cdc.gov/healthyschools/physicalactivity/pdf/17 278143-A PE-PA-Framework 508.pdf 
5. Bauman AE, Grunseit AC, Rangul V, Heitmann BL. Physical activity, obesity and mortality: Does pattern of physical activity have stronger epidemiological associations? BMC Public Health. 2017; 17(1):1-12.

6. Medina, C., Jáuregui, A., Campos-Nonato, I., \& Barquera, S. Prevalence and trends of physical activity in children and adolescents: Results of the Ensanut 2012 and Ensanut MC 2016. Salud Publica de Mexico, 2018; 60(3), 263-271. https://doi. org/10.21149/8819

7. Instituto Nacional de Salud Pública (INSP), "Encuesta de salud y nutrición de medio camino", 2016, Recueprado de: https://www.gob.mx/cms/uploads/attachment/file/209093/ENSANUT.pdf

8. Shamah-Levy T, Vielma-Orozco E, Heredia-Hernández $O$, Romero-Martínez $M$, Mojica-Cuevas J, Cuevas-Nasu L, Santaella-Castell JA, Rivera-Dommarco J. Encuesta Nacional de Salud y Nutrición 2018-19: Resultados Nacionales. Cuernavaca, México: Instituto Nacional de Salud Pública, 2020, Disponible en: https://ensanut. insp.mx/encuestas/ensanut2018/informes.php

9. Hall-López JA, Ochoa-Martínez PY, Meza F, Sánchez R, Sáenz-López P. Comparación de la actividad física por género y grasa corporal en escolares mexicanos. Rev Iberoam Ciencias la Act Física y el Deport. 2019; 8(1):1.

10. Amireault $S$, Godin G. The godin-shephard leisure-time physical activity questionnaire: Validity evidence supporting its use for classifying healthy adults into active and insufficiently active categories. Percept Mot Skills. 2015; 120 (2):604-22.

11. National Physical Activity Plan Alliance (NAPAP). The 2018 United States Report Card on Physical Activity for Children and Youth. Washington, DC: National Physical Activity Plan Alliance, 2018. Available form: https://physicalactivityplan.org/projects/PA/2018/2018 USReportCard UPDATE 12062018.pdf?pdf=page-link

12. Pereira SA, Seabra AT, Silva RG, Zhu W, Beunen GP, Maia JA. Correlates of health-related physical fitness levels of Portuguese children. Int J Pediatr Obes. 2011; 6 (1):53-9.

13. São João TM, Rodrigues RCM, Gallani MCBJ, Miura CTP, Domingues G de BL, Amireault $S$, et al. Validade da versão brasileira do Godin-Shephard Leisure-Time Physical Activity Questionnaire. Cad Saude Pública. 2015; 31(9):1825-38.

14. Godin G. The Godin-Shephard Leisure-Time Physical Activity Questionnaire. Heal Fit J Canada. 2011; 4 (1):18-22.

15. The International Diabetes Federation (IDF) consensus definition of the METABOLIC in Children. Pediatr Diabetes. 2007; 1-24.

16. Food and Nutrition Technical Assistance III (FANTA III). BMI and BMI-for-Age LookUp Tables for Children and Adolescents 5 - 18 Years of Age and BMI Look-Up Tables for Non-Pregnant, Non-Lactating Adults $\geq 19$ Years of Age. 2013; (January):1-11

17. Alonso FJ, Carranza MD, Rueda JD, Naranjo J. Composición corporal en escolares de primaria y su relación con el hábito nutricional y la práctica reglada de actividad deportiva. Rev Andaluza Med del Deport [Internet]. 2014; 7(4):137-42. Available from: http://dx.doi.org/10.1016/i.ramd.2014.08.001 
18. García Perujo M, Carrillo López PJ. Niveles de actividad física y calidad de la dieta en escolares de Educación Primaria. Rev Iberoam Ciencias la Act Física y el Deport. 2020;9 (2):16-31.

19. Ayala-Guzmán Cl, Ramos-Ibáñez N, Ortiz-Hernández L. Accelerometry does not match with self-reported physical activity and sedentary behaviors in Mexican children. Bol Med Hosp Infant Mex. 2017;74 (4):272-81.

20. Pope Z, Chen S, Pasco D, Gao Z. Effects of Body Mass Index on Children's Physical Activity Levels in School-Based "dance Dance Revolution." Games Health J. 2016; 5(3):183-8.

21. Czyż SH, Toriola AL, Starościak W, Lewandowski M, Paul Y, Oyeyemi AL. Physical fitness, physical activity, sedentary behavior, or diet-what are the correlates of obesity in Polish school children? Int J Environ Res Public Health. 2017; 14(6).

22. Morente-Oria, H. Alternativas al entrenamiento tradicional para combatir la obesidad en escolares: entrenamiento concurrente polarizado. Revista Iberoamericana De Ciencias De La Actividad Física Y El Deporte, 2019; 8(3), 45-51. https://doi. org/10.24310/riccafd.2019.v8i3.7489

Referencias totales citadas: 22

Referencias citadas correspondientes a la Rev Ib CC Act Fis Dep: 3 